\title{
The chromatic number and the least eigenvalue of a graph
}

\author{
Yi-Zheng Fan ${ }^{1,}$, Gui-Dong Yu ${ }^{1,2}$, Yi Wang ${ }^{1}$ \\ ${ }^{1}$ School of Mathematical Sciences \\ Anhui University, Hefei 230039, P. R. China \\ fanyz@ahu.edu.cn (Y.-Z. Fan), wangy@ahu.edu.cn (Y. Wang) \\ 2. School of Mathematics \& Computation Sciences \\ Anqing Normal College, Anqing 246011, P.R.China \\ yuguid@aqtc.edu.cn (G.-D. Yu) \\ Submitted: Jun 1, 2011; Accepted: Jan 20, 2012; Published: Feb 7, 2012 \\ Mathematics Subject Classifications: 05C50, 15A18
}

\begin{abstract}
In this paper we get a structural property for a graph having the minimal least eigenvalue among all graphs of fixed order and given chromatic number, and characterize such graphs under the condition that the chromatic number is not larger than half the order of the graph. As a result, we obtain a lower bound on the least eigenvalue in terms of the chromatic number, and an upper bound on the chromatic number in terms of the least eigenvalue of a graph.
\end{abstract}

\section{Introduction}

Let $G=(V, E)$ be a simple graph of order $n$ with vertex set $V=V(G)=\left\{v_{1}, v_{2}, \ldots, v_{n}\right\}$ and edge set $E=E(G)$. The adjacency matrix of $G$ is defined to be a $(0,1)$ matrix $A(G)=\left[a_{i j}\right]$ of order $n$, where $a_{i j}=1$ if $v_{i}$ is adjacent to $v_{j}$, and $a_{i j}=0$ otherwise. The eigenvalues of $A(G)$ are called the eigenvalues of the graph $G$. Since $A(G)$ is symmetric, its eigenvalues are real and can be arranged as: $\lambda_{1}(G) \geq \lambda_{2}(G) \geq \cdots \geq \lambda_{n}(G)$.

Recall that the chromatic number of a graph $G$, denoted by $\chi(G)$, is the least number of colors assigned to the vertices of $G$ such that each pair of adjacent vertices have different colors. There is a lot of work on the connection between eigenvalues and chromatic number of a graph. Wilf [18] proved that $\chi(G) \leq 1+\lambda_{1}(G)$, with equality if and only if $G$ is a complete graph or an odd cycle. Hoffman [10] shown that $\chi(G) \geq 1+\frac{\lambda_{1}(G)}{-\lambda_{n}(G)}$. Cvetković [4] obtained that $\chi(G) \geq \frac{n}{n-\lambda_{1}(G)}$. One can also refer $[9,12]$ for other lower bounds of the chromatic number of a graph. In addition, Feng et al. [8] have characterized the unique 
graph whose spectral radius attains the maximum among all graphs of fixed order and given chromatic number.

Denote by $K_{n}, O_{n}$ a complete graph and an empty graph both of order $n$, respectively. Denote by $H_{1} \vee H_{2}$ a graph obtained from two disjoint graphs $H_{1}, H_{2}$ by joining each vertex of $H_{1}$ and each of $H_{2}$. Let $G$ be a graph of order $n$. Clearly $\chi(G)=n$ if and only if $G=K_{n} ; \chi(G)=1$ if and only if $G=O_{n}$. A graph $G$ with $\chi(G)=2$ is a bipartite graph. By [3] or [19], $K_{\lceil n / 2\rceil,\lfloor n / 2\rfloor}:=O_{\lceil n / 2\rceil} \vee O_{\lfloor n / 2\rfloor}$ is the unique graph whose least eigenvalue is minimum among all graphs (or all bipartite graphs) of order $n$.

A problem arises naturally what is the graph whose least eigenvalue is minimum among all graphs of order $n$ with fixed chromatic number $\chi \geq 3$.

From the previous work, among all graphs of fixed order and fixed parameter, such as the vertex (edge) independence number or vertex (edge) cover number [14], the number of cut vertices or cut edges $[15,16,17]$, the vertex or edge connectivity [19], the diameter [20], the graphs with minimum least eigenvalue are all bipartite. So it is necessary to discuss the non-bipartite graphs with minimum least eigenvalue. This work has been done in $[5]$.

In this paper we will give a more detailed discussion on the non-bipartite graphs with minimum least eigenvalue among graphs of order $n$ with fixed chromatic $\chi\left(3 \leq \chi \leq \frac{n}{2}\right)$, and give a characterization of such graph. Using the result we obtain a lower bound of the least eigenvalue in terms of chromatic number, and an upper bound of the chromatic number in terms of the least eigenvalue of a graph.

Other work on the least eigenvalues of graphs can be referred to Bell et.al. [1, 2], Liu et.al. [11], Petrović et.al. [13], and our workgroup [6, 7]. Through these work we can not only find the structures of graphs with extremal spectral properties but also establish some relationships between the graph parameters and the eigenvalues.

\section{Preliminaries}

Let $X=\left(X_{1}, X_{2}, \ldots, X_{n}\right)^{T} \in \mathbb{R}^{n}$, and let $G$ be a graph on vertices $v_{1}, v_{2}, \ldots, v_{n}$. Then $X$ can be considered as a function defined on $V(G)$, that is, each vertex $v_{i}$ is mapped to $X_{i}=X\left(v_{i}\right) ; X_{i}$ is called a value of the vertex $v_{i}$ given by $X$. If $U \subseteq V(G)$, denote by $X(U)$ the subvector of $X$ corresponding to the vertices of $U$.

The least eigenvalue $\lambda_{n}(G)$ is now denoted by $\lambda_{\min }(G)$, and the corresponding eigenvectors are called least vectors of $G$. One can find that

$$
X^{T} A(G) X=2 \sum_{u v \in E(G)} X(u) X(v)
$$

and $\lambda$ is an eigenvector of $G$ corresponding to an eigenvector $X$ if and only if $X \neq 0$ and for each $v \in V(G)$

$$
\lambda X(v)=\sum_{u \in N(v)} X(u)
$$


where $N(v)$ denotes the neighborhood of $v$ in $G$. In addition, for an arbitrary unit vector $X \in \mathbb{R}^{n}$,

$$
\lambda_{\min }(G) \leq X^{T} A(G) X,
$$

with equality if and only if $X$ is a least vector of $G$.

We introduce a class of graphs. Given positive integer $n, \chi$ such that $3 \leq \chi \leq n-1$, define a graph $G\left(a, a^{\prime}\right):=\left(K_{a}+O_{a^{\prime}}\right) \vee\left(K_{b}+O_{b^{\prime}}\right)$, where $1 \leq a \leq \chi-1, b=\chi-a$, $a^{\prime}, b^{\prime}$ are nonnegative integers such that $a^{\prime}+b^{\prime}=n-\chi$. Note that $\chi\left(G\left(a, a^{\prime}\right)\right)=\chi$, and $\lambda_{\min }\left(G\left(a, a^{\prime}\right)\right)<-1$ as $G\left(a, a^{\prime}\right)$ is connected and non-complete.

Let $X$ be a least vector of $G\left(a, a^{\prime}\right)$. Then $X$ has constant values, say $\alpha$ on $V\left(K_{a}\right)$, $\alpha^{\prime}$ on $V\left(O_{a^{\prime}}\right), \beta$ on $V\left(K_{b}\right)$ and $\beta^{\prime}$ on $V\left(O_{b^{\prime}}\right)$. Hence $\lambda_{\min }\left(G\left(a, a^{\prime}\right)\right)$ is the least root of following equations:

$$
\lambda \alpha=(a-1) \alpha+b \beta+b^{\prime} \beta^{\prime}, \lambda \alpha^{\prime}=b \beta+b^{\prime} \beta^{\prime}, \lambda \beta=a \alpha+a^{\prime} \alpha^{\prime}+(b-1) \beta, \lambda \beta^{\prime}=a \alpha+a^{\prime} \alpha^{\prime} .
$$

So $\lambda_{\min }\left(G\left(a, a^{\prime}\right)\right)$ is the least root of the polynomial:

$$
f\left(a, a^{\prime}, \lambda\right):=\lambda^{2}(\lambda-a+1)(\lambda-b+1)-\left[\left(b+b^{\prime}\right) \lambda-b^{\prime}(b-1)\right]\left[\left(a+a^{\prime}\right) \lambda-a^{\prime}(a-1)\right],
$$

where $b=\chi-a, b^{\prime}=n-\chi-a^{\prime}$, and $n, \chi$ are considered fixed numbers. In addition, from (2.1) we also find that $X$ contains no zero entries.

LEMma 2.1 Among all graphs of order $n$ and with chromatic number $\chi$, where $3 \leq \chi \leq$ $n-1$, if a graph $G$ is one whose least eigenvalue attains the minimum, then $G=G\left(a, a^{\prime}\right)$ for some $a$ and $a^{\prime}$.

Proof: Let $X$ be a unit least vector of $G$. Surely $x$ contains both positive and negative entries. Denote by $V_{+}=\{v \in G: X(v) \geq 0\}$ and $V_{-}=\{v \in G: X(v)<0\}$. Thus $\left(V_{+}, V_{-}\right)$forms a partition of the vertex set $V(G)$.

Let $\chi\left(G\left[V_{+}\right]\right)=\bar{a}, \chi\left(G\left[V_{-}\right]\right)=\bar{b}$. Then $\bar{a} \geq 1, \bar{b} \geq 1$ and $\bar{a}+\bar{b} \geq \chi$. Clearly the set $V_{+}$can be partitioned into $\bar{a}$ subsets $V_{+}^{1}, V_{+}^{2}, \ldots, V_{+}^{\bar{a}}$ such that each edge of $G\left[V_{+}\right]$joins two different subsets, and there exists at least one edge joining any two subsets. For each $i=1,2, \ldots, \bar{a}$, choose one vertex $v_{i} \in V_{+}^{i}$ with minimal value among all vertices in $V_{+}^{i}$. Now in the graph $G\left[V_{+}\right]$, adding all possible edges within the vertices $\left\{v_{1}, v_{2}, \ldots, v_{\bar{a}}\right\}$, and deleting all other edges, we get a graph $G_{1}=K_{\bar{a}}+O_{\bar{a}^{\prime}}$ with the same order as $G\left[V_{+}\right]$, where $\bar{a}^{\prime}=\left|V_{+}\right|-\bar{a}$. One can find

$$
X\left(V_{+}\right)^{T} A\left(G\left[V_{+}\right]\right) X\left(V_{+}\right) \geq X\left(V_{+}\right)^{T} A\left(G_{1}\right) X\left(V_{+}\right) .
$$

Similarly, we can get a graph $G_{2}=K_{\bar{b}}+O_{\bar{b}^{\prime}}$ with the same order as $G\left[V_{-}\right]$, where $\bar{b}^{\prime}=\left|V_{-}\right|-\bar{b}$, which holds

$$
X\left(V_{-}\right)^{T} A\left(G\left[V_{-}\right]\right) X\left(V_{-}\right) \geq X\left(V_{-}\right)^{T} A\left(G_{2}\right) X\left(V_{-}\right) .
$$

Let $G^{\prime}=G_{1} \vee G_{2}$. Then $\chi\left(G^{\prime}\right)=\bar{a}+\bar{b}$, and $X^{T} A(G) X \geq X^{T} A\left(G^{\prime}\right) X$. Now choose two positive integers $a, b$ such that $1 \leq a \leq \bar{a}, 1 \leq b \leq \bar{b}$ and $a+b=\chi$. Arbitrarily choosing 
a complete subgraph $K_{a}$ of $K_{\bar{a}}$ (respectively, $K_{b}$ of $K_{\bar{b}}$ ) and deleting all other edges in $G_{1}$ (respectively, $\left.G_{2}\right)$, we get a graph $G^{\prime \prime}=\left(K_{a}+O_{a^{\prime}}\right) \vee\left(K_{b}+O_{b^{\prime}}\right)$, where $a+a^{\prime}, b+b^{\prime}$ are the orders of $G_{1}, G_{2}$ respectively. Then $\chi\left(G^{\prime \prime}\right)=\chi$, and

$$
\lambda_{\min }(G)=X^{T} A(G) X \geq X^{T} A\left(G^{\prime}\right) X \geq X^{T} A\left(G^{\prime \prime}\right) X \geq \lambda_{\min }\left(G^{\prime \prime}\right) .
$$

By the definition of $G, \lambda_{\min }(G)=\lambda_{\min }\left(G^{\prime \prime}\right)$ and the above inequalities hold as equalities. Hence $X$ is also a least vector of $G^{\prime \prime}$. Thus $X$ contains no zero entries by the discussion prior to this lemma. By the equality $X^{T} A(G) X=X^{T} A\left(G^{\prime \prime}\right) X$, we get $G=\left(K_{a}+O_{a^{\prime}}\right) \vee$ $\left(K_{b}+O_{b^{\prime}}\right)$, as desired.

\section{Results}

Lemma 2.1 is very useful to establish a lower bound of the least eigenvalue of a graph in terms of chromatic number, and an upper bound of the chromatic number in terms of least eigenvalue. Returning to (2.2), we temporarily assume that $a, a^{\prime}$ are real variables of $f\left(a, a^{\prime}, \lambda\right)$, and will show that for an arbitrary given $\lambda \in\left(-\frac{n}{2},-1\right)$, and given $\chi \leq \frac{n}{2}$,

$$
f\left(a, a^{\prime}, \lambda\right) \geq f\left(\frac{\chi}{2}, \frac{n-\chi}{2}, \lambda\right)
$$

with equality if and only if $a=\frac{\chi}{2}$ and $a^{\prime}=\frac{n-\chi}{2}$. This implies the least root of $f\left(\frac{\chi}{2}, \frac{n-\chi}{2}, \lambda\right)$ is not greater than that of $f\left(a, a^{\prime}, \lambda\right)$.

Assume that $\lambda \in\left(-\frac{n}{2},-1\right)$ and $\chi \leq \frac{n}{2}$ in the following. Without loss of generality, assume $a \geq b$. Taking derivative of $f$ with respect to $a, a^{\prime}$ respectively, we have

$$
\begin{aligned}
& \frac{\partial f}{\partial a}=(a-b)\left[a^{\prime} b^{\prime}-\lambda\left(a^{\prime}+b^{\prime}\right)\right]+\left(a^{\prime}-b^{\prime}\right) \lambda(\lambda+1) . \\
& \frac{\partial f}{\partial a^{\prime}}=(a-b) \lambda(\lambda+1)+(\lambda-a+1)(\lambda-b+1)\left(a^{\prime}-b^{\prime}\right) .
\end{aligned}
$$

If $a^{\prime} \geq b^{\prime}$ or equivalently $a^{\prime} \geq \frac{n-\chi}{2}$, then

$$
\frac{\partial f}{\partial a} \geq 0, \quad \frac{\partial f}{\partial a^{\prime}} \geq 0
$$

and hence $f\left(a, a^{\prime}, \lambda\right) \geq f\left(\frac{\chi}{2}, \frac{n-\chi}{2}, \lambda\right)$.

Now assume $a^{\prime}<b^{\prime}$. If $b^{\prime}-a^{\prime} \geq a-b$, then

$$
\frac{\partial f}{\partial a^{\prime}} \leq 0
$$

and hence $f\left(a, a^{\prime}, \lambda\right) \geq f\left(a, a^{\prime}+\gamma, \lambda\right)$, where $\gamma=\frac{\left(b^{\prime}-a^{\prime}\right)-(a-b)}{2}$.

If $b^{\prime}-a^{\prime} \leq a-b$, then

$$
\frac{\partial f}{\partial a} \geq\left(b^{\prime}-a^{\prime}\right)\left[a^{\prime} b^{\prime}-\lambda\left(a^{\prime}+b^{\prime}+1+\lambda\right)\right] \geq 0
$$


since $-\frac{n}{2}<\lambda<-1$ and $a^{\prime}+b^{\prime}+1+\lambda=n-\chi+1+\lambda>\frac{n}{2}+1-\frac{n}{2}>0$. Hence $f\left(a, a^{\prime}, \lambda\right) \geq$ $f\left(a-\eta, a^{\prime}, \lambda\right)$, where $\eta=\frac{(a-b)-\left(b^{\prime}-a^{\prime}\right)}{2}$. So in both cases we have $f\left(a, a^{\prime}, \lambda\right) \geq f\left(\bar{a}, \bar{a}^{\prime}, \lambda\right)$ for some $\bar{a}$ and $\bar{a}^{\prime}$ such that $\bar{a}+\bar{a}^{\prime}=\frac{n}{2}$.

Define

$$
\varphi(\delta):=f\left(a-\delta, a^{\prime}+\delta, \lambda\right)
$$

where $\delta \in\left[0, \frac{a-b}{2}\right]$ and $a+a^{\prime}=\frac{n}{2}$. Then by the derivatives of $f$ with respect of $a, a^{\prime}$ and the chain rule of composite function,

$\varphi^{\prime}(\delta)=-(a-b-2 \delta)\left\{\left(a^{\prime}+\delta\right)\left(b^{\prime}-\delta\right)-\frac{n}{2} \lambda-2 \lambda(\lambda+1)+[\lambda+1-(a-\delta)][\lambda+1-(b+\delta)]\right\} \leq 0$.

So, if $a-b>0$, or equivalently $a>\frac{\chi}{2}$, noting $a-b=b^{\prime}-a^{\prime}$,

$$
f\left(a, a^{\prime}, \lambda\right)=\varphi(0)>\varphi\left(a-\frac{a-b}{2}, a^{\prime}+\frac{a-b}{2}\right)=f\left(\frac{\chi}{2}, \frac{n-\chi}{2}, \lambda\right) .
$$

Now taking $a=b=\frac{\chi}{2}, a^{\prime}=b^{\prime}=\frac{n-\chi}{2}$ into (2.2), we get

$$
\lambda^{2}+\left(a^{\prime}+1\right) \lambda-a^{\prime}(a-1)=0,
$$

and the least root of the equation (3.5) is

$$
\xi:=-\frac{n-\chi+2+\sqrt{(n-\chi-2)^{2}+4 \chi(n-\chi)}}{4} .
$$

If $\chi \geq 2$, then $\xi=\xi(\chi)$, as a function of $\chi$, is strictly increasing on $\chi$, and hence $-1=\xi(n) \geq \xi \geq \xi(2)=-\frac{n}{2}$. Thus we get a sharper lower bound of the least eigenvalue of a graph in terms of the chromatic number.

TheOREM 3.1 Let $G$ be a graph of order $n$ with chromatic number $\chi\left(3 \leq \chi \leq \frac{n}{2}\right)$. Then

$$
\lambda_{\min }(G) \geq-\frac{n-\chi+2+\sqrt{(n-\chi-2)^{2}+4 \chi(n-\chi)}}{4},
$$

with equality if and only if $n, \chi$ are both even and $G=G(\chi / 2,(n-\chi) / 2)$.

Replacing $a^{\prime}$ by $\frac{n}{2}-a, \lambda$ by $\xi$, rewrite (3.5) as follows:

$$
a^{2}-\left(\frac{n}{2}+1+\xi\right) a+(\xi+1)\left(\xi+\frac{n}{2}\right)=0
$$

Hence

$$
\chi=2 a=\left(\frac{n}{2}+1+\xi\right)+\sqrt{\left(\frac{n}{2}+1+\xi\right)^{2}-4(\xi+1)\left(\xi+\frac{n}{2}\right)} .
$$

Noting that $\chi=\chi(\xi)$ is also increasing on $\xi, 2=\chi\left(-\frac{n}{2}\right) \leq \chi \leq \chi(-1)=n$. For a graph $G$ with chromatic number $\chi\left(3 \leq \chi \leq \frac{n}{2}\right), \lambda_{\min }(G) \geq \xi$ by Theorem 3.1. So we get an upper bound of the chromatic number of a graph in terms of the least eigenvalue. 
TheOREM 3.2 Let $G$ be a graph of order $n$ with chromatic number $\chi\left(3 \leq \chi \leq \frac{n}{2}\right)$ and least eigenvalue $\lambda=\lambda_{\min }(G)$. Then

$$
\chi \leq\left(\frac{n}{2}+1+\lambda\right)+\sqrt{\left(\frac{n}{2}+1+\lambda\right)^{2}-4(\lambda+1)\left(\lambda+\frac{n}{2}\right)},
$$

with equality if and only if $n, \chi$ are both even and $G=G(\chi / 2,(n-\chi) / 2)$.

At the end of this paper, we characterize the unique graph whose least eigenvalue attains the minimum among all graphs with fixed order and chromatic number not greater than half of the order.

TheOREM 3.3 Among all graphs of order $n$ and with chromatic number $\chi$, where $3 \leq$ $\chi \leq \frac{n}{2}$, the graph $G(\lceil\chi / 2\rceil,\lfloor(n-\chi) / 2\rfloor)$ is the unique one whose least eigenvalue attains the minimum.

Proof: By Lemma 2.1, it is enough to consider the graph $G\left(a, a^{\prime}\right)$. Without loss of generality, we assume $a \geq b$ or equivalently $a \geq \frac{\chi}{2}$. Assume that $\lambda \in\left(-\frac{n}{2},-1\right)$ in the following discussion.

Case 1: $a^{\prime} \geq b^{\prime}$. By (3.1), the function $f$ defined in (2.2) is strictly increasing on $a$ and $a^{\prime}$ respectively. Note that $a, a^{\prime}$ now are integers. To make $f$ attain the minimum, $a=\lceil\chi / 2\rceil$ and $a^{\prime}=\lceil(n-\chi) / 2\rceil$. Hence, if $a \geq b+2$ or $a^{\prime} \geq b^{\prime}+2$ then $\lambda_{\min }\left(G\left(a, a^{\prime}\right)\right)>$ $\lambda_{\min }(G(\lceil\chi / 2\rceil,\lceil(n-\chi) / 2\rceil)$.

To reach the final result, we need to discuss the case of $(n-\chi)=: 2 m+1$ being odd. If $\chi$ is even, then $G(\lceil\chi / 2\rceil, m+1)$ is isomorphic to $G(\lceil\chi / 2\rceil, m)$; otherwise by a little calculation $f(\lceil\chi / 2\rceil, m+1, \lambda)-f(\lceil\chi / 2\rceil, m, \lambda)>0$, which implies that $\lambda_{\min }(G(\lceil\chi / 2\rceil, m+1))>$ $\lambda_{\min }(G(\lceil\chi / 2\rceil, m))$.

Case 2: $b^{\prime}>a^{\prime}$. If $a=b$, the result follows from Case 1 by considering the graph $G\left(b, b^{\prime}\right)$. So we assume that $a>b$ in the following.

If $b^{\prime}-a^{\prime} \geq a-b$, then $\frac{\partial f}{\partial a^{\prime}} \leq 0$ by (3.2). Hence, if $b^{\prime}-a^{\prime} \geq(a-b)+2$, then $f\left(a, a^{\prime}, \lambda\right)>f\left(a, a^{\prime}+1, \lambda\right)$. So $\lambda_{\min }\left(G\left(a, a^{\prime}\right)\right)>\lambda_{\min }\left(G\left(a, a^{\prime}+1\right)\right) \geq \cdots \geq \lambda_{\min }\left(G\left(a, a^{\prime}+l\right)\right)$, for some positive integer $l$ such that $\left(b^{\prime}-l\right)-\left(a^{\prime}+l\right)$ equals $a-b$ or $a-b+1$.

If $b^{\prime}-a^{\prime} \leq a-b$, then $\frac{\partial f}{\partial a}>0$ by (3.3). Hence, if $b^{\prime}-a^{\prime} \leq(a-b)-2$, then $f\left(a, a^{\prime}, \lambda\right)>$ $f\left(a-1, a^{\prime}, \lambda\right)$, and hence $\lambda_{\min }\left(G\left(a, a^{\prime}\right)\right)>\lambda_{\min }\left(G\left(a-1, a^{\prime}\right)\right) \geq \lambda_{\min }\left(G\left(a-k, a^{\prime}\right)\right)$ for some positive integer $k$ such that $b^{\prime}-a^{\prime}$ equals $(a-k)-(b+k)$ or $(a-k)-(b+k)-1$.

By above discussion, we only need to consider the graph $G\left(a, a^{\prime}\right)$ with $b^{\prime}-a^{\prime}$ equal to one of the values $a-b-1, a-b, a-b+1$. If $b^{\prime}-a^{\prime}=a-b+1$, by a little calculation $f\left(a, a^{\prime}, \lambda\right)>f\left(a, a^{\prime}+1, \lambda\right)$, and hence $\lambda_{\min }\left(G\left(a, a^{\prime}\right)>\lambda_{\min }\left(G\left(a, a^{\prime}+1\right)\right.\right.$. So it is enough to deal with the first two types of graphs $G\left(a, a^{\prime}\right)$.

If $b^{\prime}-a^{\prime}=a-b=: \zeta \geq 2$, using the function $\varphi(\delta)$ defined in (3.4),

$$
f\left(a, a^{\prime}, \lambda\right)=\varphi(0)>\phi\left(a-\lfloor\zeta / 2\rfloor, a^{\prime}+\lfloor\zeta / 2\rfloor\right)=f\left(a-\lfloor\zeta / 2\rfloor, a^{\prime}+\lfloor\zeta / 2\rfloor, \lambda\right),
$$

and hence $\lambda_{\min }\left(G\left(a, a^{\prime}\right)\right)>\lambda_{\min }\left(G\left(a-\lfloor\zeta / 2\rfloor, a^{\prime}+\lfloor\zeta / 2\rfloor\right)\right)=\lambda_{\min }(G(\lceil\chi / 2\rceil,\lfloor(n-\chi) / 2\rfloor))$. 
If $b^{\prime}-a^{\prime}=(a-b)-1$ and $a-b \geq 2$, then $f\left(a, a^{\prime}, \lambda\right)-f\left(a-1, a^{\prime}+1, \lambda\right)=$

$$
\left(-\lambda^{2}-2 b^{\prime} \lambda+a^{\prime} b^{\prime}\right)+(a-b-2)\left[-\lambda^{2}-2\left(a^{\prime}+a-1\right) \lambda+a^{\prime} b^{\prime}+(a-2) b\right]>0,
$$

where the first summand is positive as $2 b^{\prime}>a^{\prime}+b^{\prime}=n-\chi \geq \frac{n}{2}$, and the second is nonnegative as $2\left(a+a^{\prime}-1\right)=2\left(b+b^{\prime}\right)=n-1>\frac{n}{2}$. So $\lambda_{\min }\left(G\left(a, a^{\prime}\right)>\lambda_{\min }\left(G\left(a-1, a^{\prime}+1\right)\right)\right.$. The result follows by repeated using this inequality.

Remark: For the graph $G(\chi / 2,(n-\chi) / 2)$, where $n, \chi(\chi \leq n / 2)$ are both even, the spectral radius is also the largest root of the $f(\chi / 2,(n-\chi) / 2, \lambda)$, and is given by

$$
\lambda_{1}(G(\chi / 2,(n-\chi) / 2))=\frac{2 a-1+a^{\prime}+\sqrt{(2 a-1)^{2}+a^{\prime}\left(a^{\prime}+2\right)}}{2}>2 a-1+\frac{a^{\prime}}{2},
$$

where $a=\chi / 2, a^{\prime}=(n-\chi) / 2$. According to Wilf's bound,

$$
\chi(G(\chi / 2,(n-\chi) / 2))=\chi<\chi+\frac{n-\chi}{4}=1+\lambda_{1}(G(\chi / 2,(n-\chi) / 2)) .
$$

However, our bound in Theorem 3.2 for this graph is tight. For an odd cycle, Wilf's bound is tight but our bound is not good.

Theorems 3.1-3.3 deal with the graphs $G$ of order $n$ with $3 \leq \chi(G) \leq n / 2$. By a number of numerical experiments on small graphs, we conjecture that they hold for all graphs $G$ without limitation on the range of $\chi(G)$.

Acknowledgments. This work was supported by National Natural Science Foundation of China (11071002), Program for New Century Excellent Talents in University, Key Project of Chinese Ministry of Education (210091), Specialized Research Fund for the Doctoral Program of Higher Education (20103401110002), Science and Technological Fund of Anhui Province for Outstanding Youth (10040606Y33), NSF of Department of Education of Anhui Province (KJ2011A195, KJ2010B136), Project of Anhui Province for Excellent Young Talents in Universities (2009SQRZ017ZD), Scientific Research Fund for Fostering Distinguished Young Scholars of Anhui University(KJJQ1001), Academic Innovation Team of Anhui University Project (KJTD001B), Fund for Youth Scientific Research of Anhui University (KJQN1003), and Innovation Fund for Graduates of Anhui University.

\section{References}

[1] F. K. Bell, D. Cvetković, P. Rowlinson, S. K. Simić, Graph for which the least eigenvalues is minimal, I, Linear Algebra Appl., 429(2008), 234-241.

[2] F. K. Bell, D. Cvetković, P. Rowlinson, S. K. Simić, Graph for which the least eigenvalues is minimal, II, Linear Algebra Appl., 429(2008), 2168-2179.

[3] G. Constantine, Lower bounds on the spectra of symmetric matrices with nonnegative entries, Linear Algebra Appl., 65(1985), 171-178.

[4] D. Cvetković, Chromatic number and the spectrum of a graph, Publ. Inst. Math. (Beograd), 14(28)(1972), 25-38. 
[5] Y.-Z. Fan, The least eigenvalue of a nonbipartite graph, J. Anqing Teachers College (Nat. Sci. Ed.) (in Chinese), 15(3)(2009), 1-3.

[6] Y.-Z. Fan, Y. Wang, Y.-B. Gao, Minimizing the least eigenvalues of unicyclic graphs with application to spectral spread, Linear Algebra Appl., 429(2-3)(2008), 577-588.

[7] Y.-Z. Fan, F.-F. Zhang, Y. Wang, The least eigenvalue of the complements of trees, Linear Algebra Appl., doi:10.1016/j.laa.2011.04.011.

[8] L. Feng, Q. Li, X.-D. Zhang, Spectral radii of graphs with given chromatic number, Appl. Math. Lett., 20(2007), 158-162.

[9] W. H. Haemers, Interlacing eigenvalues and graphs, Linear Algebra Appl., 227/228(1995), 593-616.

[10] A. J. Hoffman, On eigenvalues and colourings of graphs, in Graph Theory and its Applications (B. Harris, Ed.), Academic Press, New York, 1970, pp. 79-91.

[11] R. Liu, M. Zhai, J. Shu, The least eigenvalue of unicyclic graphs with $n$ vertices and $k$ pendant vertices, Linear Algebra Appl., 431(5-7)(2009), 657-665.

[12] V. Nikiforov, Chromatic number and spectral radius, Linear Algebra Appl., 426(2007), 810-814.

[13] M. Petrović, B. Borovićanin, T. Aleksić, Bicyclic graphs for which the least eigenvalue is minimum, Linear Algebra Appl., 430(4)(2009), 1328-1335.

[14] Y.-Y. Tan, Y.-Z. Fan, The vertex (edge) independence number, vertex (edge) cover number and the least eigenvalue of a graph, Linear Algebra Appl., 433 (2010), 790-795.

[15] Y. Wang, Y.-Z. Fan, On the least eigenvalue of graphs with cut vertices, J. Math. Res. Expo., 30(6)(2010), 951-956.

[16] Y. Wang, Y.-Z. Fan, The least eigenvalue of a graph with cut vertices, Linear Algebra Appl., 433(2010), 19-27.

[17] Y. Wang, Y.-Z. Fan, The least eigenvalue of a graph with cut edges, Graph \& Comb., doi: 10.1007/s00373-011-1060-z.

[18] H. S. Wilf, The eigenvalues of a graph and its chromatic number, J. London Math. Soc., 42 (1967), 330-332.

[19] M.-L. Ye, Y.-Z. Fan, D. Liang, The least eigenvalue of graphs with given connectivity, Linear Algebra Appl., 430(4)(2009), 1375-1379.

[20] M. Zhai, R. Liu, J. Shu, Minimizing the least eigenvalue of unicyclic graphs with fixed diameter, Discrete Math., 310 (2010), 947-955. 